\title{
PLANS FOR A 750 MEV ELECTRON BEAM TEST FACILITY AT FERMILAB*
}

\author{
M. Church ${ }^{\#}$, S. Nagaitsev, FNAL, Batavia, IL 60510, U.S.A. \\ P. Piot, FNAL and Northern Illinois Univ., DeKalb, IL 60115, U.S.A.
}

\begin{abstract}
A $750 \mathrm{MeV}$ electron beam test facility at Fermilab is in the planning and early construction phase. An existing building is being converted for this facility. The photoinjector currently in use at the Fermilab NICADD Photoinjector Laboratory (FNPL) will be moved to the new facility and upgraded to serve as an injector for a beam acceleration section consisting of three TTF or ILCtype RF cryomodules. A low energy off-axis beamline will be constructed to test ILC crab cavity designs and provide opportunities for other tests. Downstream beamlines will consist of a diagnostic section, a beam test area for additional beam experiments, and high power beam dumps. The initial program for this facility will concentrate on testing ILC-type cryomodules and RF control with full ILC beam intensity. A future building expansion will open up further possibilities for beam physics and beam technology experiments.
\end{abstract}

\section{INTRODUCTION}

The Fermilab ILC Test Accelerator at the New Muon Lab (ILCTA) is part of a larger effort at Fermilab to develop the infrastructure to build, test, and certify ILCtype RF cryomodules and associated diagnostics. The overall layout of the ILCTA injector, acceleration section (with two RF cryomodules), and high energy beamlines is shown in Figures 1-3. This facility will eventually have 3 consecutive RF cryomodules powered by a single modulator/klystron in a configuration identical to that envisioned for the ILC. It will provide a system test, with beam, of a complete ILC "RF unit" for both high level and low level RF control including high intensity beam feedback systems. In particular, in order to meet the ILC luminosity goal, the requirement on RF cavity phase stability is $0.25^{\circ} \mathrm{RMS}$ at $1.3 \mathrm{GHz}$, and the requirement on RF cavity amplitude stability is $0.5 \%$ RMS. These are very challenging requirements, and an RF system test with full beam intensity will be required to verify them.

The injector will be capable of providing ILCintensity beam $(3.2 \mathrm{nC} /$ bunch, 3000 bunches/pulse, 5 pulses/sec) at $45 \mathrm{MeV}$ to the acceleration section. RMS bunch lengths of less than 300 microns will be obtainable. At an average acceleration gradient of $31.5 \mathrm{MeV} /$ meter, the beam can be accelerated to over $800 \mathrm{MeV}$ by the three RF cryomodules. Diagnostics in both the injector and the high energy beamlines will be able to fully characterize the beam properties before and after the acceleration

\footnotetext{
*Work supported by Fermi Research Alliance LLC. Under DE-AC0207CH11359 with the U.S. DOE

\# church@fnal.gov
}

section. Either of two $50 \mathrm{~kW}$ beam dumps will safely absorb the beam. There are "free" sections in both the low energy and high energy beamlines for test and development of new instrumentation.

ILCTA will serve as an effective training ground for both accelerator staff and students in the field of high intensity electron beams and superconducting RF. It will also serve as a first stage in developing a facility for advanced accelerator R\&D at Fermilab.

\section{INJECTOR}

The L-band electron gun currently under design will be similar to the gun now in use at the photoinjector test facility at DESY in Zeuthen (PITZ). The laser system currently in use at FNPL [1] will be used at ILCTA. A more detailed description of the gun and modifications planned for ILCTA are described in another paper in this conference [2]. A dark current kicker and a dark current collimator will follow immediately downstream of the gun in order to suppress dark current from the gun photocathode. The collimator is followed by two 9-cell superconducting L-band RF "capture" cavities operating at average accelerating gradients up to $\sim 10 \mathrm{MeV} / \mathrm{m}$ and $\sim 30 \mathrm{MeV} / \mathrm{m}$. The downstream end of the 2 nd capture cavity is $7.1 \mathrm{~m}$ downstream of the photocathode. Eventually a 3rd harmonic $(3.9 \mathrm{GHz}) \mathrm{RF}$ cavity will be installed downstream of the 2nd capture cavity in order to linearize the longitudinal bunch rotation and enable the injector to produce bunches with RMS bunch length less than 300 microns.

Extensive beam simulations for this part of the injector have been done with ASTRA [3]. Based on these simulations, for ILC beam intensities $(3.2 \mathrm{nC} /$ bunch $)$ we expect normalized transverse emittance of $\sim 4.5 \mathrm{E}-6$ at 45 $\mathrm{MeV}$. This emittance is acceptable for cleanly transporting beam downstream to the beam dumps and for performing critical RF tests.

The precise layout of the injector downstream of the 2nd RF cavity has not yet been finalized. Approximately 11 quadrupoles of the type currently in use at FNPL will provide beam focusing. An additional 3 skew quadrupoles will be used to produce flat beams [4]. A four-magnet chicane will complete the longitudinal bunch rotation initiated by the 2 capture cavities and 3rd harmonic cavity. This chicane will also be used for precise beam energy and energy width measurements. A dogleg consisting of several dipoles downstream of the chicane can be used to steer the beam into a parallel beamline, to be used for testing ILC crab cavity designs and other components. The total length is 22.3 meters. 


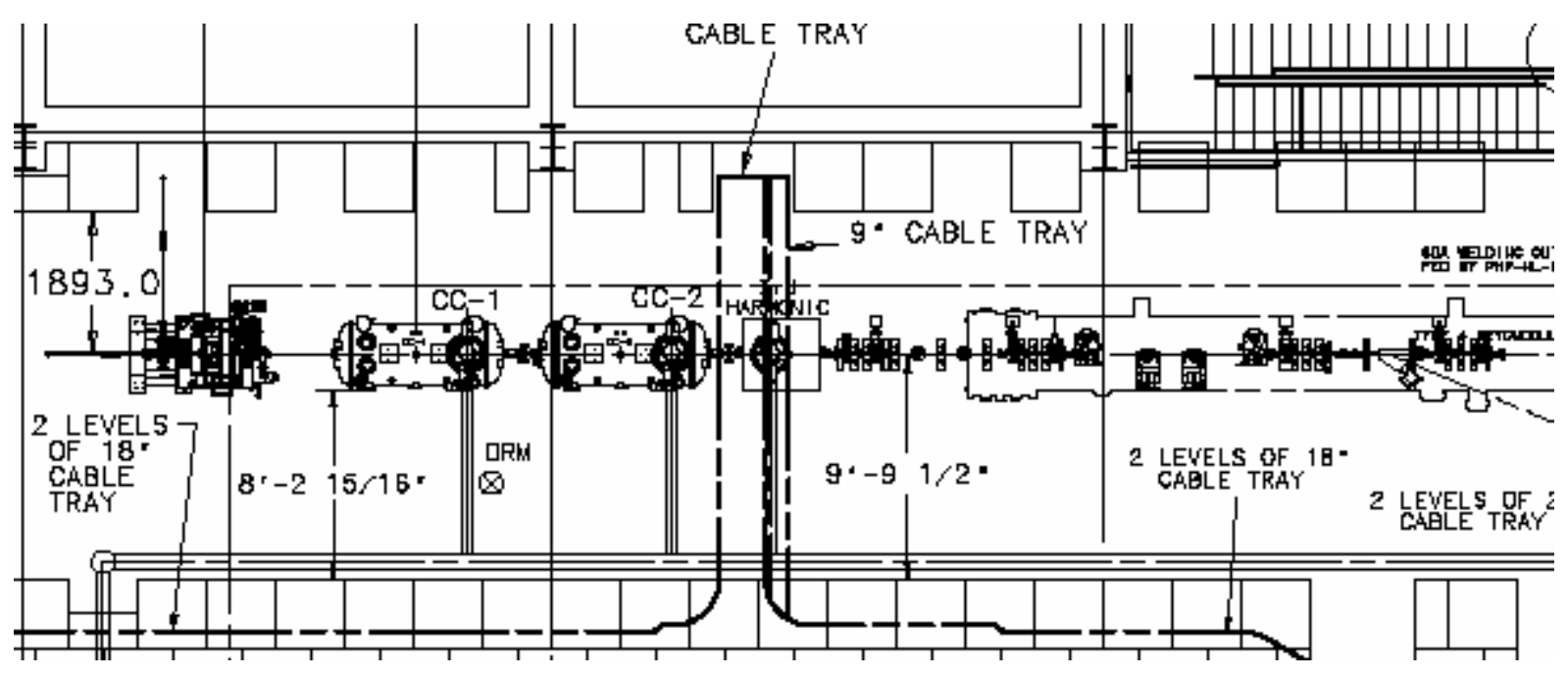

Figure 1: Injector for ILCTA. Electron gun is on the left.

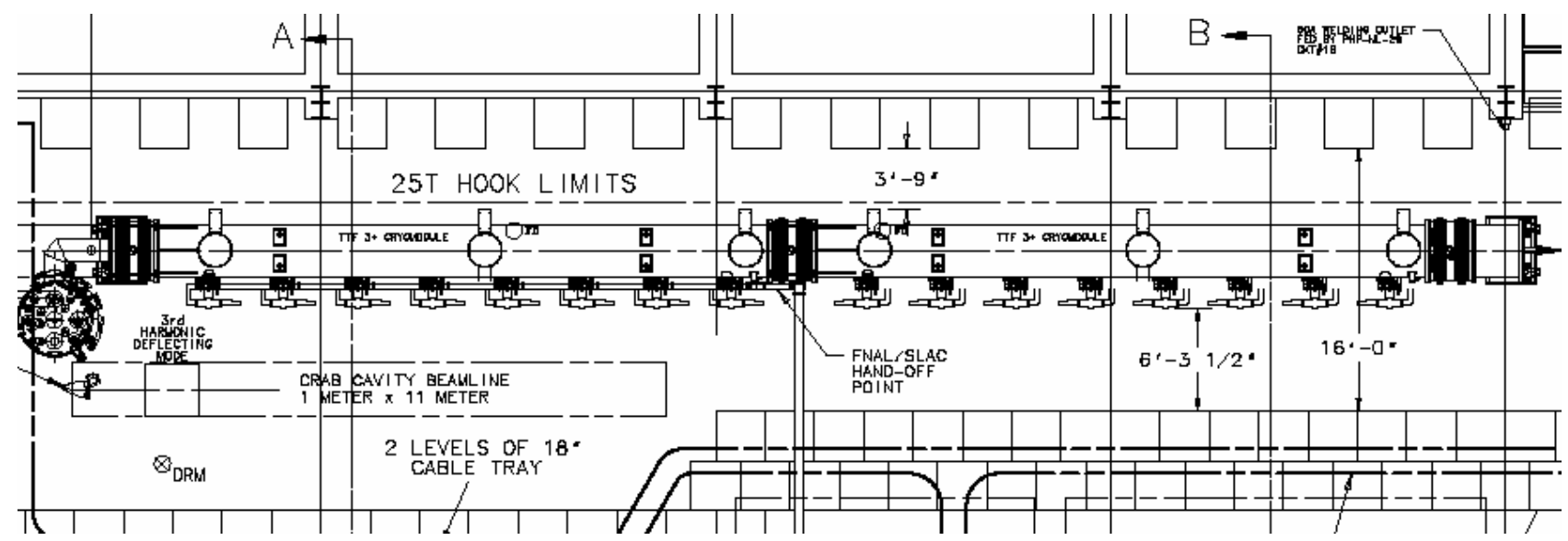

Figure 2: Two RF cryomodule acceleration section for ILCTA.

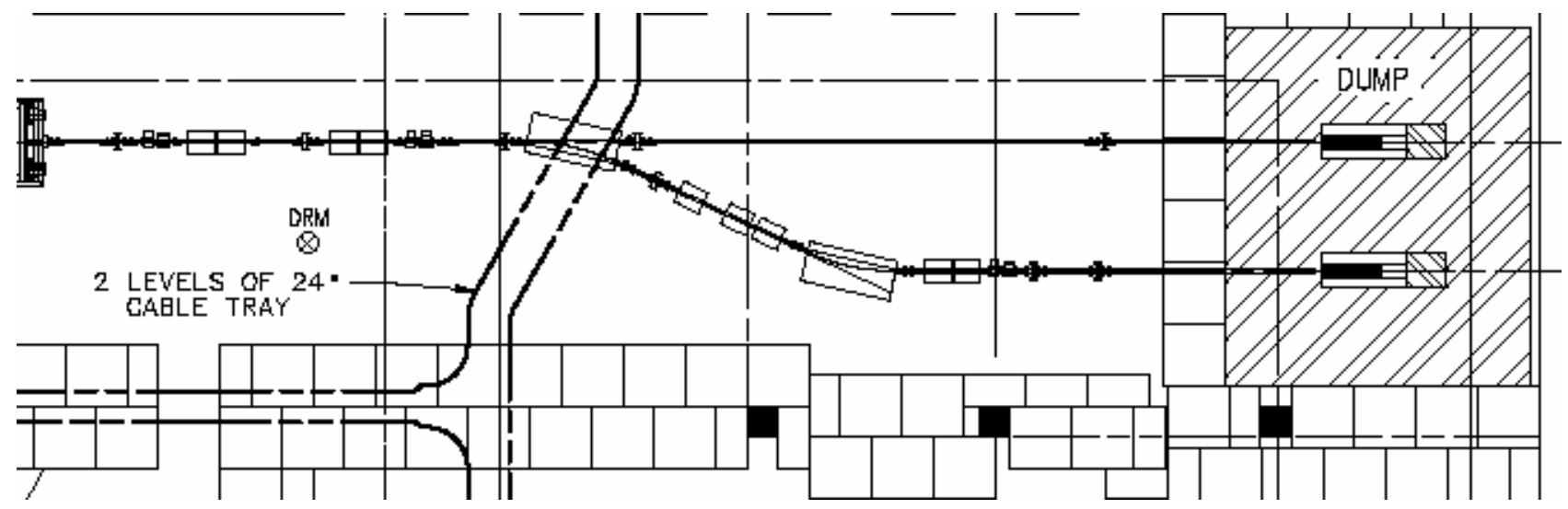

Figure 3: High energy beamlines for ILCTA 


\section{ACCELERATION SECTION}

The construction of the acceleration section will be staged. Stage 1 will consist of one TTF type $3+R F$ cryomodule, stage 2 will add an additional TTF type $3+$ cryomodule, and stage 3 will add an ILC type 4 RF cryomodule. The cave construction will be such that cryomodules are easily replaceable with more advanced cryomodules. RF cryomodules will be powered by a single modulator/klystron.

\section{HIGH ENERGY BEAMLINES}

The high energy beamlines must provide enough diagnostics to fully characterize the beam, transport beam cleanly to the dump, and provide space for the installation and test of new diagnostics. The beamline section immediately downstream of the last RF cryomodule will have four quadrupoles to match lattice functions to the downstream spectrometer, dipole steerers to correct for any steering errors originating in the acceleration section, and drift spaces for ample diagnostics. Diagnostics are button-type BPM's, vacuum crosses to house optical transition radiation detectors (OTRs) for beam size measurements, a current monitor, beam phase monitor, and bunch length monitor.

The spectrometer consists of a 25 deg dipole magnet followed by a dispersive section and instrumentation. Beam energy and beam energy width will be measured using BPMs and OTRs before and after this 1 st $25 \mathrm{deg}$ dipole. Three quadrupoles serve to cancel the dispersion and control the beta functions coming out of the 2 nd 25 deg bend magnet.

The straight-through beamline is left open at this time and is intended to accomodate instrumentation for R\&D.

Both beam dumps must be able to safely absorbe $50 \mathrm{kw}$ of beam power. The core of the dump will be very similar to the TTF1 dump core. It consists of a graphite cylinder surrounded by an aluminum tube and water cooling lines. Downstream of the graphite core is a copper plug to absorb some of the beam energy before it escapes from the dump. This core is surrounded by steel and concrete. The dump core will operate in atmosphere and the beamline will be capped with a Be window. Two quadrupoles in front of each dump will serve to blow up the transverse beam size to greater than $3 \mathrm{~mm}$ RMS in both dimensions, in order to prevent damage to the Be window or graphite dump core. Extensive energy deposition, heating, and radiation calculations have been done on both the Be window and the dump core, and are reported in another paper in this conference [5]. The overall length of the downstream beamline is $\sim 18$ meters.

\section{TENTATIVE SCHEDULE}

Extensive upgrades to building infrastructure started in 2006. Currently a cryogenic system with a single Tevatron-style satellite refrigerator with the capacity to service the photoinjector and a single RF cryomodule is nearing completion. A 2nd satellite refrigerator will be added by Summer of 2008 to service a 2nd RF cryomodule. In order to provide enough cooling capacity for 3 RF cryomodules operating at full ILC gradient and full beam current, these 2 satellite refrigerators will be replaced by a single larger refrigeration system. Low conductivity water systems, electrical power, building environmental controls, radiation shielding, alignment monuments, electronics racks and cable trays, and control rooms are currently under development. One of the injector RF capture cavities is now under test and will be installed at ILCTA in the Summer of 2007. The photoinjector at FNPL, including the other RF capture cavity, will be disassembled and moved to ILCTA starting in early 2008. The new electron gun will be installed at ILCTA starting in Spring 2008. We expect to receive the 1 st TTF type $3+$ cryomodule for installation at ILCTA by Fall 2007, the 2nd TTF type 3+ cryomodule by Summer 2008, and the 3rd cryomodule, ILC type 4, by Spring 2009.

In order to install the 3rd RF cryomodule an extension of the ILCTA facility will be built. This will be accomplished with minimal disruption to ongoing operations at ILCTA by building a new building just downstream of the current ILCTA building and then connecting the two buildings with a tunnel. The new building will have additional space for beam test areas.

\section{REFERENCES}

[1] J. Li, R. Tikhoplav, A. Melissinos, "Performance of the upgraded laser system for the Fermilab-NIU photoinjector", NIM A, V564, p57, 2006

[2] S. Nagaitsev, et. al., "Fermilab L-band Electron Gun for the ILC Cryomodule Test Facility", PAC07, Albuquerque, NM, June 2007

[3] K. Floettman, http://www.desy.de/ mpyflo/

[4] P. Piot, Y.-E. Sun, K.-J. Kim, "Photoinjectorgeneration of a flat electron beam with transverse emittance ratio of 100", Phys Rev. ST Accel. Beams, V9, \#3, 2006

[5] M. Church, et. al. "Modeling and Design of the ILC Test Area Beam Absorbers at Fermilab", PAC07, Albuquerque, NM, June 2007 